\title{
Comparison Effect of Nebulized Milrinone and Intravenous Milrinone in Patients With Pulmonary Hypertension Who Candidate of Open-heart Surgery: a Double Blind Randomized Clinical Trial
}

\section{Sara Joreirahmadi}

Ahvaz Jundishapur University of Medical Sciences: Ahvaz Jondishapour University of Medical Sciences

Fatemeh Javaherforoosh zadeh ( $\sim \mathrm{f}$ _javaherforoosh@yahoo.com )

Ahvaz Jondishapour University of Medical Sciences https://orcid.org/0000-0002-7687-5888

Marziyeh Babazadehdezfuli

Shiraz Medical School: Shiraz University of Medical Sciences

Behnam Gholizadeh

Ahvaz Jundishapur University of Medical Sciences: Ahvaz Jondishapour University of Medical Sciences Nima Bakhtiari

Ahvaz Jundishapur University of Medical Sciences: Ahvaz Jondishapour University of Medical Sciences

\section{Research article}

Keywords: Valvular heart disease, Milrinone, pulmonary hypertension, cardiopulmonary bypass

Posted Date: August 17th, 2021

DOI: https://doi.org/10.21203/rs.3.rs-764237/v1

License: (c) (i) This work is licensed under a Creative Commons Attribution 4.0 International License.

Read Full License 


\section{Abstract}

Objective: This study was aimed to compare the effect of nebulized milrinone versus intravenous administration of milrinone in pulmonary hypertension patients who are candidates for open-heart surgery

Method: A total of 32 patients candidates for open-heart surgery complicated by pulmonary hypertension were involved in this study and were arbitrarily classified into the nebulized milrinone group $(n=16)$ and intravenous milrinone group $(n=16)$ that they received drug after the opening of the cross-clamp of the aorta and before weaning off CPB.

Heart rate (HR), cardiac output (CO), cardiac index (Cl), stork volume (SV), systolic blood pressure (SBP), diastolic blood pressure (DBP), mean arterial pressure (MAP), central venous pressure (CVP), mean pulmonary artery pressure (mPAP), Systemic vascular resistance (SVR) pulmonary vascular resistance(PVR) and MAP/mPAP ratio, Extubation time, ICU stay and hospital stay were collected.

Results: MAP and MAP/mPAP ratio increased significantly, however, mPAP was significantly lower in the nebulized milrinone group than in the intravenous milrinone group. Extubation time, ICU stay and hospital stays decreased significantly in the nebulized milrinone group than in the intravenous milrinone group.

Conclusion: the administration of the nebulized milrinone before weaning off from CPB, accelerated and facilitated separation from CPB as it significantly decreases mPAP and preserves MAP.

Trial registry: Registered under No. IRCT20201104049266N1

\section{Introduction}

Pulmonary hypertension $(\mathrm{PH})$ is a progressive sickness caused by heart, lung, or systemic diseases. Regardless of $\mathrm{PH}$ etiology, it is associated with increased patient morbidity and mortality (1). PH is more common in young adults 30-40 years old and women are more common than men. This pathologic disorder in progressive conditions leads to right-sided heart failure and severe dyspnea (2). PH is divided into primary and secondary categories. Primary $\mathrm{PH}$ is an infrequent condition and its incidence is estimated at 2 to 3 per million people. It occurs in children and women is three times more common than in men (3).

One of the phosphodiesterase inhibitors and indicators, that has been widely using in open cardiac surgery for the management of pulmonary hypertension $(\mathrm{PH})$ is milrinone. It is usually given by intravenous administration, principally during challenging weaning off from cardiopulmonary bypass (CPB)(4). This inhibitory effect is associated with increased intracellular ionized calcium and contractile strength of heart muscle(4). A significant disadvantage of intravenous administration of milrinone is systemic hypotension(5). To prevent the complication of hypotension caused by this method, inhalation or nebulization has been projected in place of another therapeutic way for the administration of this 
inodilator drug (5). In this route of drug administration rapid absorption, high bioavailability, and high local concentrations of the drug are achieved (6). A protective effect against increased $\mathrm{PH}$ was achieved in patients undergoing on-pump cardiac surgery by Inhaled milrinone $(6,7)$. This effect was done via lessening CPB-related inflammation(8), avoiding pulmonary endothelial dysfunction(9), and enabling weaning off from $\mathrm{CPB}(10)$. A randomized controlled trial recently established the beneficial effect of inhaled milrinone in reducing the amount of pulmonary hypertension; but, this is not very effective in separating from CPB, and prevention of right ventricular dysfunction (RV) has not yet been determined. The cause of these results can be due to the low doses of the drug delivery (11) this study aimed to compare the effect of nebulized milrinone with intravenous administration of milrinone in patients with pulmonary hypertension who are candidates for open-heart surgery.

\section{Methods}

\section{Study design and Ethical statement:}

This study is a double-blind clinical trial. The study was permitted by the Anesthesiology and Pain Research Center, Ahvaz Jundishapur University of Medical Sciences, Ahvaz, Iran

(IR.AJUMS.REC.1399.654). The written informed agreement was achieved from all patients participating in the trial. The samples are all patients who underwent open-heart surgery in the Golestan hospital, Ahvaz, Iran. From October 2020 to July 2021 that meets suitability criteria.

\section{Participant}

Thirty-two patients candidates for open-heart surgery were selected. The inclusion criteria were as follows: (1) age 20 to 70 years, (2) on pump cardiac surgery; (3) pulmonary arterial pressure more than 40 $\mathrm{mmHg}$ that was evaluated by transthoracic and transesophageal echocardiography. The exclusion criteria included the following: (1) Patients were indisposed to the participant or were incompetent to communicate, (2) Redo surgery, (3) emergency surgery, (4) history of chronic obstructive pulmonary disease (COPD), (5) history of severe hepatic or renal dysfunction, (6) hemoptysis. Consort flow diagram

\section{Randomization and Blindness:}

The patients were randomized using the simple incidental method, after recognizing appropriate individuals, they were randomly distributed a three-digit exclusive code using the random table. The final digit on the right concludes the patient group. If the figure is $0,1,2,3,4$ it was located in the nebulized milrinone (Group $A, N=16$ ), and if the figure is $5,6,7,8,9$ it was situated in the intravenous milrinone (Group $B, N=16$ ). To ensure that, the patients, the surgeon, and the investigators were unaware of the treatment group before the study begins and they were uninformed of the type of administration of the drug and the surgeries performed by the same surgeon.

\section{Sample size:}


Based on a previous study (12), the prevalence $(P)$ of this disorder is estimated to be 0.1 . Therefore, according to the accuracy of the study $(d=0.1)$, the significance level of $95 \%$ in this study and the error level (alpha) of the average are considered 0.05. Z with $95 \%$ confidence is 1.96 the prevalence equation of sample size detection 16 samples were selected in each group.

\section{Standardized anesthesia}

Premedication was managed according to local practices $(0.1 \mathrm{mg} / \mathrm{kg}$ of Morphine and $0.025 \mathrm{mg} / \mathrm{kg}$ of Diazepam as IM). Standard monitoring was performed after entering the operating room, such as electrocardiogram, heart rate (HR), non-invasive blood pressure (NIBP), invasive blood pressure (by radial artery cannulation), and pulse oximetry $\left(\mathrm{SpO}_{2}\right)$. The anesthesia procedure, the surgeon, and the cardiopulmonary bypass technique was intended to be identical for all patients. General anesthesia was induced with $0.1-0.2 \mathrm{mg} / \mathrm{kg}$ of midazolam (Caspian Tamin, Iran), $0.5-1 \mu \mathrm{g} / \mathrm{kg}$ of sufentanil (Aburaihan, Iran), $0.1 \mathrm{mg} / \mathrm{kg}$ of Etomidate (Lipuro, B. Braun, India), and $0.5 \mathrm{mg} / \mathrm{kg}$ of cisatracurium (Rosamed, Iran). Additionally, isoflurane $1 \%$ (Piramal Critical Care, USA) in $50 \%$ oxygen, $0.1 \mu \mathrm{g} / \mathrm{kg} / \mathrm{h}$ of sufentanil, 0.1 $\mathrm{mg} / \mathrm{kg} / \mathrm{h}$ of midazolam, and $0.1 \mathrm{mg} / \mathrm{kg} / \mathrm{h}$ of cisatracurium were used to maintain general anesthesia. End-tidal $\mathrm{CO}_{2}$ was retained in the range of $30-40 \mathrm{mmHg}$, and arterial $\mathrm{pH}$ was maintained at $7.4 \pm 0.02$ for all patients in both groups. After induction of anesthesia, pulmonary artery catheters are inserted from the right internal jugular vein.

\section{Intervention}

After surgical repair of the cardiac defect and the opening of the cross-clamp of the aorta, and before weaning off from CPB; Group A patients received nebulized milrinone (Milrinone Lactate $1 \mathrm{mg} \cdot \mathrm{ml}^{-1}$; Pharmaceutical Partners of Canada Inc., Richmond Hill) through a jet nebulizer (Ref 8901; Salter Labs, Arvin, CA) attached to the inspiratory limb of the ventilator with a bypass flow of oxygen at $10 \mathrm{~L} / \mathrm{min}$ (50$80 \mathrm{\mu g} \mathrm{kg}^{-1}$ ) close the endotracheal tube, dissolved in $5 \mathrm{~mL}$ normal saline. Patients in group B received intravenous bolus milrinone $50 \mathrm{\mu g} \mathrm{kg}^{-1}$, followed by continuous administration of $0.5 \mu \mathrm{g} \mathrm{kg}^{-1} \mathrm{~min}^{-1}$, started immediately before trying to weaning off from CPB. After administration of the drug (nebulized or IV) weaning off from CPB was started. This process was based on the CVP protocol. (13) Before CPB weaning off, dobutamine was infused at a dose of $5 \mathrm{\mu g} \mathrm{kg}^{-1} \mathrm{~min}^{-1}$ that was augmented up to $10 \mathrm{\mu g} \mathrm{kg}^{-1}$ $\mathrm{min}^{-1}$ according to patient response. Epinephrine infusion was added up to a dose of $0.05 \mu \mathrm{g} \mathrm{kg}^{-1} \mathrm{~min}^{-1}$ that was augmented up to $0.1 \mu \mathrm{g} \mathrm{kg}^{-1} \mathrm{~min}^{-1}$ according to patient response if the mean arterial blood pressure during weaning off $\mathrm{CPB}$ did not exceed $50 \mathrm{mmHg}$. After surgery, all patients were transferred to the cardiovascular intensive care unit (CV ICU) sedated and on controlled mechanical ventilation. Patients were extubated when the weaning criteria emerged.

\section{Data collection}

The measured hemodynamic parameters included heart rate (HR), cardiac output (CO), cardiac index (Cl), stork volume (SV), systolic blood pressure (SBP), diastolic blood pressure (DBP), mean arterial pressure 
(MAP), central venous pressure (CVP), mean pulmonary artery pressure (MPAP), Systemic vascular resistance (SVR) pulmonary vascular resistance(PVR) and MAP/M PAP ratio was calculated using the standard method, after induction of anesthesia as baseline (T1), 10, 30 and 60 minutes after the termination of cardiopulmonary bypass ( CPB ) (T2, T3, and T4).

\section{Statistical analysis}

All Patient characteristics data were expressed as mean \pm standard deviation (SD) and percentages. Evaluations of hemodynamic variables between groups were accomplished with the Student t-test for normally distributed variables. To evaluate variations over time within each group were used one-way analysis of variance (ANOVA) on repeated measurements. Two-way analysis of covariance (ANCOVA) determined for baseline values (T1) was used to compare groups at T2, T3, and T4. Statistical analyses were done with the computer software IBM SPSS Statistics for Windows, Version 19.0 (Armonk, NY: IBM Corp). P-value less than 0.05 was considered as statistical significance.

\section{Results}

A total of 32 patients were employed over the study period. The patients were categorized into two groups, each of them included 16 patients, inhaled milrinone group and IV milrinone. Regarding demographic data (Table 1), there were no statistically significant differences between both studied groups.

For all patients, the mean \pm SD age was $47 \pm 4$ years and there were 16 men and 16 women with $25 \pm 3$ as a mean \pm SD of BMI score. A total of 8 complex procedures were performed. The before surgery mPAP $(43.38 \pm 6.32$ vs. $53.25 \pm 10.50 \mathrm{mmHg}, p=0.09)$ were higher in the IV group compared to the Milerinone group. 
Table 1

Baseline Appearances of the Study Population Characteristic

\begin{tabular}{|c|c|c|}
\hline Characteristic & $\begin{array}{l}\text { Nebulized milrinone group } \\
(n=16)\end{array}$ & $\begin{array}{l}\text { IV milrinone group } \\
(n=16)\end{array}$ \\
\hline Age & $46 \pm 4$ & $P=0.1705$ \\
\hline Sex (male) & $6(\% 37)$ & $10(\%$ 62) \\
\hline BMI $\left(\mathrm{kg} / \mathrm{m}^{2}\right)$ & $25 \pm 3$ & $P=0.6749$ \\
\hline Smoking & $2(\% 12)$ & $6(\% 37)$ \\
\hline \multicolumn{3}{|l|}{ NYHA class } \\
\hline 1 & 0 & 0 \\
\hline 2 & $12(\% 75)$ & $12(\% 75)$ \\
\hline 3 & $4(\% 25)$ & $4(\% 25)$ \\
\hline 4 & 0 & 0 \\
\hline \multicolumn{3}{|l|}{ Type of surgery } \\
\hline Isolated valve & $4(\% 25)$ & $6(\% 37.5)$ \\
\hline Multiple valve & $2(\% 12.5)$ & $4(\% 25)$ \\
\hline Complex & $6(\% 37.5)$ & $2(\% 12.5)$ \\
\hline Other & $4(\% 25)$ & $4(\% 25)$ \\
\hline \multicolumn{3}{|l|}{ Comorbidities } \\
\hline Hypertension & 0 & $6(\% 37.5)$ \\
\hline Diabetes mellitus & 0 & $6(\% 37.5)$ \\
\hline COPD & 0 & $4(\% 25)$ \\
\hline Coronary artery disease & 0 & $2(\% 12.5)$ \\
\hline Hyperlipidemia & $6(\% 37.5)$ & $10(\%$ 62.5) \\
\hline hypothyroidism & $4(\% 25)$ & 0 \\
\hline No comorbidities & $10(\%$ 62.5) & $6(\% 37.5)$ \\
\hline
\end{tabular}

*Variables expressed as number (\%) and mean \pm standard deviation. BMI, body mass index; NYHA, New York Heart Association; COPD, chronic obstructive pulmonary disease; ACEl, angiotensinconverting enzyme inhibitor; CPB, cardiopulmonary bypass. 


\begin{tabular}{|c|c|c|c|}
\hline Characteristic & $\begin{array}{l}\text { Nebulized milrinone group } \\
(n=16)\end{array}$ & \multicolumn{2}{|c|}{$\begin{array}{l}\text { IV milrinone group } \\
(n=16)\end{array}$} \\
\hline \multicolumn{4}{|l|}{ Drug therapy at admission } \\
\hline Warfarin & 0 & \multicolumn{2}{|l|}{$2(\% 12.5)$} \\
\hline Levothyroxine & $4(\% 25)$ & \multicolumn{2}{|l|}{0} \\
\hline Beta-blockers & $4(\% 25)$ & \multicolumn{2}{|l|}{$6(\% 37.5)$} \\
\hline ACEIS & $4(\% 25)$ & \multicolumn{2}{|l|}{$8(\% 50)$} \\
\hline Digoxin & $2(\% 12.5)$ & \multicolumn{2}{|l|}{$2(\% 12.5)$} \\
\hline Diuretics & $2(\% 12.5)$ & \multicolumn{2}{|l|}{$6(\% 37.5)$} \\
\hline Salicylates & $6(\% 37.5)$ & \multicolumn{2}{|l|}{$6(\% 37.5)$} \\
\hline Statins & $2(\% 12.5)$ & \multicolumn{2}{|l|}{$4(\% 25)$} \\
\hline Metformin & 0 & \multicolumn{2}{|l|}{$2(\% 12.5)$} \\
\hline No drug therapy at admission & $4(\% 25)$ & \multicolumn{2}{|l|}{$6(\% 37.5)$} \\
\hline Left ventricular ejection fraction (\%) & $45(40-50)$ & $49(45-50)$ & $P=0.7986$ \\
\hline Duration of surgery (min) & $104 \pm 33$ & $163 \pm 38$ & $P=0.072$ \\
\hline \multicolumn{3}{|l|}{ Aorta clamping } & $P=0.205$ \\
\hline $\begin{array}{l}\text { *Variables expressed as number (\%) } \\
\text { New York Heart Association; COPD, ch } \\
\text { converting enzyme inhibitor; CPB, car }\end{array}$ & $\begin{array}{l}\text { mean } \pm \text { standard deviation. } \\
\text { ic obstructive pulmonary dis } \\
\text { ulmonary bypass. }\end{array}$ & $\begin{array}{l}\text { body mass ir } \\
\text { e; ACEl, angio }\end{array}$ & $\begin{array}{l}\text { ex; NYHA, } \\
\text { nsin- }\end{array}$ \\
\hline
\end{tabular}

\section{Within Groups Comparison}

Hemodynamic variables are shown in Table 2 for the IV and nebulized group separately. There were no variations over time for CVP, SV and $\mathrm{Cl}$ in the both group $(p>0.05)$ but there were changes in nebulized and IV group over time for $\mathrm{HR}(\mathrm{P}<0.0001$ vs $\mathrm{P}=0.02)$, mPAP $(\mathrm{P}=0.001$ vs $\mathrm{P}<0.0001)$, MAP/mPAP $(P=$ 0.0032 vs $P<0.0008)$ and $P V R(P<0.0001$ in both group). There were changes over time for $C O(p=$ $0.01), \operatorname{SVR}(p=0.005), \operatorname{SBP}(p=0.04), \operatorname{DBP}(p=0.04)$ and MAP $(p=0.01)$, in patients receiving nebulized milerinone. 
Table 2

Hemodynamic variables within groups

\begin{tabular}{|c|c|c|c|c|c|c|}
\hline Variables & Groups & $\begin{array}{l}\text { T1 } \\
\text { (Baseline) }\end{array}$ & $\begin{array}{l}\text { T2 } \\
\text { (10 min } \\
\text { after CPB } \\
\text { off) }\end{array}$ & $\begin{array}{l}\text { T3 } \\
\text { (30 min } \\
\text { after CPB } \\
\text { off) }\end{array}$ & $\begin{array}{l}\text { T4 } \\
\text { (60 min } \\
\text { after CPB } \\
\text { off) }\end{array}$ & $P$-Value \\
\hline \multirow[t]{2}{*}{ HR (beats/min) } & Nebulized & $\begin{array}{l}71.25 \pm \\
10.01\end{array}$ & $\begin{array}{l}97.75 \pm \\
9.453\end{array}$ & $\begin{array}{l}99.88 \pm \\
12.37\end{array}$ & $\begin{array}{l}98.38 \pm \\
14.32\end{array}$ & $\begin{array}{l}< \\
0.0001^{1}\end{array}$ \\
\hline & IV & $\begin{array}{l}73.50 \pm \\
11.72\end{array}$ & $\begin{array}{l}93.13 \pm \\
11.74\end{array}$ & $\begin{array}{l}93.38 \pm \\
9.13\end{array}$ & $\begin{array}{l}98.20 \pm \\
9.60\end{array}$ & $0.002^{2}$ \\
\hline \multirow[t]{2}{*}{ CVP(mm Hg) } & Nebulized & $\begin{array}{l}11.75 \pm \\
3.69\end{array}$ & $\begin{array}{l}12.75 \pm \\
3.45\end{array}$ & $11.25 \pm 2.6$ & $12.5 \pm 4.07$ & 0.38 \\
\hline & IV & $\begin{array}{l}10.88 \pm \\
2.74\end{array}$ & $\begin{array}{l}12.38 \pm \\
3.06\end{array}$ & $\begin{array}{l}13.31 \pm \\
3.27\end{array}$ & $\begin{array}{l}13.75 \pm \\
2.98\end{array}$ & 0.55 \\
\hline \multirow[t]{2}{*}{ CO (L/min) } & Nebulized & $\begin{array}{l}4.213 \pm \\
1.49\end{array}$ & $4.92 \pm 1.02$ & $5.90 \pm 1.65$ & $6.11 \pm 1.32$ & $0.01^{3}$ \\
\hline & IV & $\begin{array}{l}4.40 \pm \\
1.51\end{array}$ & $4.87 \pm 1.03$ & $5.55 \pm 1.32$ & $5.65 \pm 1.36$ & 0.19 \\
\hline \multirow[t]{2}{*}{$\begin{array}{l}\text { SVR } \\
\text { (dynes.sec.cm-5) }\end{array}$} & Nebulized & $\begin{array}{l}1261 \pm \\
438.3\end{array}$ & $\begin{array}{l}838.3 \pm \\
161.8\end{array}$ & $\begin{array}{l}841.8 \pm \\
230.5\end{array}$ & $\begin{array}{l}850.7 \pm \\
30.28\end{array}$ & $0.005^{4}$ \\
\hline & IV & $\begin{array}{l}1216 \pm \\
451.5\end{array}$ & $\begin{array}{l}897.9 \pm \\
381.6\end{array}$ & $\begin{array}{l}806.1 \pm \\
269.6\end{array}$ & $\begin{array}{l}900.9 \pm \\
101.8\end{array}$ & 0.19 \\
\hline \multirow[t]{2}{*}{$\begin{array}{l}\text { Stroke volume } \\
(\mathrm{mL})\end{array}$} & Nebulized & $\begin{array}{l}49.83 \pm \\
9.152\end{array}$ & $\begin{array}{l}53.33 \pm \\
7.11\end{array}$ & $\begin{array}{l}60.50 \pm \\
16.98\end{array}$ & $\begin{array}{l}59.88 \pm \\
12.98\end{array}$ & 0.36 \\
\hline & IV & $\begin{array}{l}56.14 \pm \\
16.27\end{array}$ & $\begin{array}{l}50.57 \pm \\
6.47\end{array}$ & $\begin{array}{l}59.13 \pm \\
15.15\end{array}$ & $\begin{array}{l}65.67 \pm \\
13.14\end{array}$ & 0.34 \\
\hline \multirow[t]{2}{*}{$\mathrm{Cl}$} & Nebulized & $\begin{array}{l}2.37 \pm \\
0.74\end{array}$ & $2.81 \pm 0.69$ & $3.32 \pm 1.07$ & $3.4 \pm 0.72$ & 0.07 \\
\hline & IV & $\begin{array}{l}2.55 \pm \\
0.80\end{array}$ & $2.97 \pm 0.73$ & $3.23 \pm 0.69$ & $3.38 \pm 0.86$ & 0.20 \\
\hline \multirow[t]{2}{*}{$\mathrm{SBP}(\mathrm{mm} \mathrm{Hg})$} & Nebulized & $\begin{array}{l}103.0 \pm \\
4.41\end{array}$ & $\begin{array}{l}85.00 \pm \\
17.94\end{array}$ & $\begin{array}{l}97.38 \pm \\
17.12\end{array}$ & $\begin{array}{l}108.8 \pm \\
8.84\end{array}$ & $0.004^{5}$ \\
\hline & IV & $\begin{array}{l}98.50 \pm \\
10.20\end{array}$ & $\begin{array}{l}82.88 \pm \\
19.53\end{array}$ & $\begin{array}{l}86.00 \pm \\
10.78\end{array}$ & $\begin{array}{l}98.00 \pm \\
16.72\end{array}$ & 0.07 \\
\hline \multirow[t]{2}{*}{$\mathrm{DBP}(\mathrm{mm} \mathrm{Hg})$} & Nebulized & $\begin{array}{l}61.75 \pm \\
14.65\end{array}$ & $\begin{array}{l}50.13 \pm \\
8.37\end{array}$ & $\begin{array}{l}56.13 \pm \\
12.32\end{array}$ & $\begin{array}{l}64.00 \pm \\
9.18\end{array}$ & $0.004^{6}$ \\
\hline & IV & $\begin{array}{l}55.88 \pm \\
7.06\end{array}$ & $\begin{array}{l}47.63 \pm \\
11.36\end{array}$ & $\begin{array}{l}48.63 \pm \\
8.36\end{array}$ & $\begin{array}{l}55.88 \pm \\
9.59\end{array}$ & 0.13 \\
\hline
\end{tabular}




\begin{tabular}{|c|c|c|c|c|c|c|}
\hline Variables & Groups & $\begin{array}{l}\text { T1 } \\
\text { (Baseline) }\end{array}$ & $\begin{array}{l}\text { T2 } \\
\text { (10 min } \\
\text { after CPB } \\
\text { off) }\end{array}$ & $\begin{array}{l}\text { T3 } \\
\text { (30 min } \\
\text { after CPB } \\
\text { off) }\end{array}$ & $\begin{array}{l}\text { T4 } \\
\text { ( } 60 \text { min } \\
\text { after CPB } \\
\text { off) }\end{array}$ & $P$-Value \\
\hline \multirow[t]{2}{*}{$\mathrm{MAP}(\mathrm{mm} \mathrm{Hg})$} & Nebulized & $\begin{array}{l}74.13 \pm \\
11.80\end{array}$ & $\begin{array}{l}61.13 \pm \\
9.90\end{array}$ & $\begin{array}{l}67.88 \pm \\
14.04\end{array}$ & $\begin{array}{l}77.75 \pm \\
9.93\end{array}$ & $0.01^{7}$ \\
\hline & IV & $\begin{array}{l}69.13 \pm \\
4.85\end{array}$ & $\begin{array}{l}57.00 \pm \\
11.53\end{array}$ & $\begin{array}{l}58.88 \pm \\
7.53\end{array}$ & $\begin{array}{l}68.25 \pm \\
11.97\end{array}$ & 0.06 \\
\hline \multirow[t]{2}{*}{ MPAP $(\mathrm{mm} \mathrm{Hg})$} & Nebulized & $\begin{array}{l}43.38 \pm \\
6.32\end{array}$ & $\begin{array}{l}35.63 \pm \\
9.03\end{array}$ & $\begin{array}{l}33.13 \pm \\
8.42\end{array}$ & $\begin{array}{l}32.50 \pm \\
8.86\end{array}$ & $0.001^{8}$ \\
\hline & IV & $\begin{array}{l}52.00 \pm \\
9.81\end{array}$ & $\begin{array}{l}36.88 \pm \\
13.6\end{array}$ & $\begin{array}{l}34.38 \pm \\
10.50\end{array}$ & $\begin{array}{l}32.50 \pm \\
11.34\end{array}$ & $\begin{array}{l}< \\
0.0001^{9}\end{array}$ \\
\hline \multirow[t]{2}{*}{$\mathrm{MAP} / \mathrm{mPAP}$} & Nebulized & $\begin{array}{l}1.72 \pm \\
0.30\end{array}$ & $1.79 \pm 0.42$ & $2.18 \pm 0.69$ & $2.56 \pm 0.76$ & $0.026^{10}$ \\
\hline & IV & $\begin{array}{l}1.35 \pm \\
0.19\end{array}$ & $1.67 \pm 0.56$ & $1.84 \pm 0.50$ & $2.19 \pm 0.42$ & $0.007^{11}$ \\
\hline \multirow[t]{2}{*}{$\begin{array}{l}\text { PVR(dynes.sec.cm- } \\
\text { 5) }\end{array}$} & Nebulized & $\begin{array}{l}301.5 \pm \\
4.62\end{array}$ & $\begin{array}{l}258.9 \pm \\
13.71\end{array}$ & $\begin{array}{l}242.1 \pm \\
6.77\end{array}$ & $\begin{array}{l}165.1 \pm \\
6.51\end{array}$ & $\begin{array}{l}< \\
0.0001^{12}\end{array}$ \\
\hline & IV & $\begin{array}{l}302.6 \pm \\
9.07\end{array}$ & $\begin{array}{l}219.4 \pm \\
5.42\end{array}$ & $\begin{array}{l}241.8 \pm \\
6.13\end{array}$ & $\begin{array}{l}151.4 \pm \\
6.98\end{array}$ & $\begin{array}{l}< \\
0.0001^{13}\end{array}$ \\
\hline \multicolumn{7}{|c|}{$\begin{array}{l}\text { One-way repeated ANOVA *Variables expressed as adjusted mean } \pm \text { standard deviation. T1: after } \\
\text { induction of anesthesia as Baseline, T2: } 10 \text { min after CPB off, T3: } 30 \text { minutes after CPB off, T4: } 60 \\
\text { min after CPB off. ANOVA, analysis of variance; HR, Heart rate; CVP, central venous pressure; CO, } \\
\text { cardiac output; SVR, systemic vascular resistance; SV, stroke volume; Cl, cardiac index; SBP, systolic } \\
\text { blood pressure; DBP, diastolic blood pressure; MAP, mean arterial pressure; SPAP, systolic pulmonary } \\
\text { artery pressure; }{ }^{1} \mathrm{HR} \text { became higher in the nebulized group in T4 compared to T1, in T3 compared with } \\
\text { T1 and in T2 compared with T1. }{ }^{2} \mathrm{HR} \text { increased in the IV group in T4 compared to T1, in T3 compared } \\
\text { with T1, and in T2 compared with T1. }{ }^{3} \mathrm{CO} \text { in the nebulized group became higher at T4 compared to } \\
\text { T1. }{ }^{4} \mathrm{SVR} \text { in the nebulized group became decrease in T4 compared to T1, in T3 compared with T1, and } \\
\text { in T2 compared with T1. }{ }^{5} \mathrm{SBP} \text { in the nebulized group became higher at T4 compared to T2. }{ }^{6} \mathrm{DBP} \\
\text { increase in the nebulized group in T4 compares to T2. }{ }^{7} \mathrm{MAP} \text { decrease in the nebulized group in T2 } \\
\text { compare to T1 and became higher at T4 compared to T3 and T2. }{ }^{8} \mathrm{MPAP} \text { in the nebulized group lower } \\
\text { at T4 and T3 compared to T1. }{ }^{9} \mathrm{MPAP} \text { in the IV group decrease in T4 compared with T1 and T2 and in } \\
\text { T3 and T2 compared with T1. }{ }^{10} \mathrm{MAP} / \mathrm{mPAP} \text { in the Nebulized group increase in T4 compared with } \\
\mathrm{T} 1 .{ }^{11} \mathrm{MAP} / \mathrm{mPAP} \text { in the IV group increase in T4 compared with T1. }{ }^{12} \mathrm{PVR} \text { in the nebulized group } \\
\text { became higher at T1 compared to T2, T3 and T4 and lower in T4 compared to T3 and T2. }{ }^{13} \mathrm{PVR} \text { in } \\
\text { the IV group became higher at T1 compared to T2, T3 and T4 and lower in T4 compared to T3, T2, } \\
\text { and T1. }\end{array}$} \\
\hline
\end{tabular}

\section{Between Groups Comparison}


Hemodynamic measurements between groups comparisons are shown in Table 3: Groups were similar at all times for all variables. However at T2 $(p=0.0074), \mathrm{T} 3(p=0.0201)$ and T4 $(p=0.0125)$, the means of the Nebulized milrinone group were higher for SBP and T2 $(p<0.0001)$ and T4 $(p=0.0032)$ for PVR. (Fig. 1) 
Table 3

Hemodynamic variables between groups

\begin{tabular}{|c|c|c|c|c|c|c|c|}
\hline \multirow[t]{2}{*}{ Variables } & \multirow[t]{2}{*}{ Group } & $\mathrm{T} 2$ & \multirow[t]{2}{*}{ P-Value } & T3 & \multirow[t]{2}{*}{ P-Value } & T4 & \multirow[t]{2}{*}{ P-Value } \\
\hline & & $\begin{array}{l}\text { (10 min } \\
\text { after CPB } \\
\text { off) }\end{array}$ & & $\begin{array}{l}\text { ( } 30 \text { min } \\
\text { after CPB } \\
\text { off) }\end{array}$ & & $\begin{array}{l}\text { (60 min } \\
\text { after } \\
\text { CPB } \\
\text { off) }\end{array}$ & \\
\hline \multirow[t]{2}{*}{$\begin{array}{l}\text { HR } \\
\text { (beats/min) }\end{array}$} & Nebulized & $\begin{array}{l}97.75 \pm \\
9.453\end{array}$ & \multirow[t]{2}{*}{0.2073} & $\begin{array}{l}99.88 \pm \\
12.37\end{array}$ & \multirow[t]{2}{*}{0.1519} & $\begin{array}{l}98.38 \pm \\
14.32\end{array}$ & \multirow[t]{2}{*}{0.6919} \\
\hline & IV & $\begin{array}{l}93.13 \pm \\
11.74\end{array}$ & & $\begin{array}{l}93.38 \pm \\
9.13\end{array}$ & & $\begin{array}{l}98.20 \pm \\
9.60\end{array}$ & \\
\hline \multirow[t]{2}{*}{ CVP $(\mathrm{mm} \mathrm{Hg})$} & nebulized & $\begin{array}{l}12.75 \pm \\
3.45\end{array}$ & \multirow[t]{2}{*}{0.7892} & $\begin{array}{l}93.38 \pm \\
9.13\end{array}$ & \multirow[t]{2}{*}{0.0947} & $\begin{array}{l}12.5 \pm \\
4.07\end{array}$ & \multirow[t]{2}{*}{0.2800} \\
\hline & IV & $\begin{array}{l}12.38 \pm \\
3.06\end{array}$ & & $\begin{array}{l}11.25 \pm \\
2.6\end{array}$ & & $\begin{array}{l}13.75 \pm \\
2.98\end{array}$ & \\
\hline \multirow[t]{2}{*}{$\mathrm{CO}(\mathrm{L} / \mathrm{min})$} & nebulized & $\begin{array}{l}4.92 \pm \\
1.02\end{array}$ & \multirow[t]{2}{*}{0.7493} & $\begin{array}{l}5.90 \pm \\
1.65\end{array}$ & \multirow[t]{2}{*}{0.4759} & $\begin{array}{l}6.11 \pm \\
1.32\end{array}$ & \multirow[t]{2}{*}{0.4149} \\
\hline & IV & $\begin{array}{l}4.87 \pm \\
1.03\end{array}$ & & $\begin{array}{l}5.55 \pm \\
1.32\end{array}$ & & $\begin{array}{l}5.65 \pm \\
1.36\end{array}$ & \\
\hline \multirow{2}{*}{$\begin{array}{l}\text { SVR } \\
\text { (dynes.sec.cm- } \\
\text { 5) }\end{array}$} & nebulized & $\begin{array}{l}838.3 \pm \\
161.8\end{array}$ & \multirow[t]{2}{*}{0.6093} & $\begin{array}{l}841.8 \pm \\
230.5\end{array}$ & \multirow[t]{2}{*}{0.9617} & $\begin{array}{l}850.7 \pm \\
30.28\end{array}$ & \multirow[t]{2}{*}{0.5679} \\
\hline & IV & $\begin{array}{l}897.9 \pm \\
381.6\end{array}$ & & $\begin{array}{l}806.1 \pm \\
269.6\end{array}$ & & $\begin{array}{l}900.9 \pm \\
101.8\end{array}$ & \\
\hline \multirow[t]{2}{*}{$\begin{array}{l}\text { Stroke volume } \\
(\mathrm{mL})\end{array}$} & nebulized & $\begin{array}{l}53.33 \pm \\
7.11\end{array}$ & \multirow[t]{2}{*}{0.1436} & $\begin{array}{l}60.50 \pm \\
16.98\end{array}$ & \multirow[t]{2}{*}{0.5194} & $\begin{array}{l}59.88 \pm \\
12.98\end{array}$ & \multirow[t]{2}{*}{0.7202} \\
\hline & IV & $\begin{array}{l}50.57 \pm \\
6.47\end{array}$ & & $\begin{array}{l}59.13 \pm \\
15.15\end{array}$ & & $\begin{array}{l}65.67 \pm \\
13.14\end{array}$ & \\
\hline \multirow[t]{2}{*}{$\mathrm{Cl}\left(\mathrm{L} / \mathrm{min} / \mathrm{m}^{2}\right)$} & nebulized & $2.81 \pm 0.69$ & \multirow[t]{2}{*}{0.9612} & $\begin{array}{l}3.32 \pm \\
1.07\end{array}$ & \multirow[t]{2}{*}{0.5694} & $\begin{array}{l}3.4 \pm \\
0.72\end{array}$ & \multirow[t]{2}{*}{0.6480} \\
\hline & IV & $\begin{array}{l}2.97 \pm \\
0.73\end{array}$ & & $\begin{array}{l}3.23 \pm \\
0.69\end{array}$ & & $\begin{array}{l}3.38 \pm \\
0.86\end{array}$ & \\
\hline \multirow[t]{2}{*}{$\mathrm{SBP}(\mathrm{mm} \mathrm{Hg})$} & nebulized & $\begin{array}{l}85.00 \pm \\
17.94\end{array}$ & \multirow[t]{2}{*}{$0.0009^{1}$} & $\begin{array}{l}97.38 \pm \\
17.12\end{array}$ & \multirow[t]{2}{*}{$0.0034^{2}$} & $\begin{array}{l}108.8 \pm \\
8.84\end{array}$ & \multirow[t]{2}{*}{$0.0015^{3}$} \\
\hline & IV & $\begin{array}{l}82.88 \pm \\
19.53\end{array}$ & & $\begin{array}{l}86.00 \pm \\
10.78\end{array}$ & & $\begin{array}{l}98.00 \pm \\
16.72\end{array}$ & \\
\hline
\end{tabular}

Two-way analysis of covariance (ANCOVA) *Variables presented as mean \pm standard deviation. T1: after induction of anesthesia as Base line, T2: 10 min after CPB off, T3: 30 minutes after CPB off, T4: 60 min after CPB off. ANCOVA, analysis of covariance; HR, Heart rate; CVP, central venous pressure; CO, cardiac output; SVR, systemic vascular resistance; SV, CI, SBP, DBP; MAP, mean arterial pressure; MPAP, mean pulmonary arterial pressure; ${ }^{1,2,3}$ SBP is smaller at T2 $(p=0.0009), \mathrm{T} 3(p=0.0034)$ and T4 $(p=0.0015)$ in IV group. ${ }^{4,5}$ PVR is smaller at T2 $(p<0.0001)$ and T4 $(p=0.0032)$ in IV group. 


\begin{tabular}{|c|c|c|c|c|c|c|c|}
\hline Variables & Group & $\begin{array}{l}\text { T2 } \\
\text { (10 min } \\
\text { after CPB } \\
\text { off) }\end{array}$ & P-Value & $\begin{array}{l}\text { T3 } \\
\text { (30 min } \\
\text { after CPB } \\
\text { off) }\end{array}$ & P-Value & $\begin{array}{l}\text { T4 } \\
(60 \text { min } \\
\text { after } \\
\text { CPB } \\
\text { off) }\end{array}$ & P-Value \\
\hline \multirow[t]{2}{*}{$\mathrm{DBP}(\mathrm{mm} \mathrm{Hg})$} & nebulized & $\begin{array}{l}50.13 \pm \\
8.37\end{array}$ & \multirow[t]{2}{*}{0.5903} & $\begin{array}{l}56.13 \pm \\
12.32\end{array}$ & \multirow[t]{2}{*}{0.8047} & $\begin{array}{l}64.00 \pm \\
9.18\end{array}$ & \multirow[t]{2}{*}{0.7178} \\
\hline & IV & $\begin{array}{l}47.63 \pm \\
11.36\end{array}$ & & $\begin{array}{l}48.63 \pm \\
8.36\end{array}$ & & $\begin{array}{l}55.88 \pm \\
9.59\end{array}$ & \\
\hline \multirow[t]{2}{*}{$\mathrm{MAP}(\mathrm{mm} \mathrm{Hg})$} & nebulized & $\begin{array}{l}61.13 \pm \\
9.90\end{array}$ & \multirow[t]{2}{*}{0.8539} & $\begin{array}{l}67.88 \pm \\
14.04\end{array}$ & \multirow[t]{2}{*}{0.4036} & $\begin{array}{l}77.75 \pm \\
9.93\end{array}$ & \multirow[t]{2}{*}{0.3533} \\
\hline & IV & $\begin{array}{l}57.00 \pm \\
11.53\end{array}$ & & $\begin{array}{l}58.88 \pm \\
7.53\end{array}$ & & $\begin{array}{l}68.25 \pm \\
11.97\end{array}$ & \\
\hline \multirow[t]{2}{*}{$\begin{array}{l}\text { MPAP }(\mathrm{mm} \\
\mathrm{Hg})\end{array}$} & nebulized & $\begin{array}{l}35.63 \pm \\
9.03\end{array}$ & \multirow[t]{2}{*}{0.1961} & $\begin{array}{l}33.13 \pm \\
8.42\end{array}$ & \multirow[t]{2}{*}{0.1384} & $\begin{array}{l}32.50 \pm \\
8.86\end{array}$ & \multirow[t]{2}{*}{0.0989} \\
\hline & IV & $\begin{array}{l}36.88 \pm \\
13.6\end{array}$ & & $\begin{array}{l}34.38 \pm \\
10.50\end{array}$ & & $\begin{array}{l}32.50 \pm \\
11.34\end{array}$ & \\
\hline \multirow[t]{2}{*}{$\mathrm{MAP} / \mathrm{mPAP}$} & nebulized & $\begin{array}{l}1.79 \pm \\
0.42\end{array}$ & \multirow[t]{2}{*}{0.2861} & $\begin{array}{l}2.18 \pm \\
0.69\end{array}$ & \multirow[t]{2}{*}{0.9132} & $\begin{array}{l}2.56 \pm \\
0.76\end{array}$ & \multirow[t]{2}{*}{0.9921} \\
\hline & IV & $\begin{array}{l}1.67 \pm \\
0.56\end{array}$ & & $\begin{array}{l}1.84 \pm \\
0.50\end{array}$ & & $\begin{array}{l}2.19 \pm \\
0.42\end{array}$ & \\
\hline \multirow{2}{*}{$\begin{array}{l}\text { PVR } \\
\text { (dynes.sec.cm- } \\
\text { 5) }\end{array}$} & nebulized & $\begin{array}{l}258.9 \pm \\
13.71\end{array}$ & \multirow[t]{2}{*}{$\begin{array}{l}< \\
0.0001^{4}\end{array}$} & $\begin{array}{l}242.1 \pm \\
6.77\end{array}$ & \multirow[t]{2}{*}{0.7460} & $\begin{array}{l}165.1 \pm \\
6.51\end{array}$ & \multirow[t]{2}{*}{$0.0032^{5}$} \\
\hline & IV & $\begin{array}{l}219.4 \pm \\
5.42\end{array}$ & & $\begin{array}{l}241.8 \pm \\
6.13\end{array}$ & & $\begin{array}{l}151.4 \pm \\
6.98\end{array}$ & \\
\hline \multicolumn{8}{|c|}{$\begin{array}{l}\text { Two-way analysis of covariance (ANCOVA) *Variables presented as mean } \pm \text { standard deviation. T1: } \\
\text { after induction of anesthesia as Base line, T2: } 10 \text { min after CPB off, T3: } 30 \text { minutes after CPB off, T4: } \\
60 \text { min after CPB off. ANCOVA, analysis of covariance; HR, Heart rate; CVP, central venous pressure; } \\
\text { CO, cardiac output; SVR, systemic vascular resistance; SV, CI, SBP, DBP; MAP, mean arterial pressure; } \\
\text { MPAP, mean pulmonary arterial pressure; } 1,2,3 \text { SBP is smaller at T2 }(p=0.0009), T 3(p=0.0034) \text { and } \\
\text { T4 }(p=0.0015) \text { in IV group. }{ }^{4,5} \text { PVR is smaller at T2 }(p<0.0001) \text { and T4 }(p=0.0032) \text { in IV group. }\end{array}$} \\
\hline
\end{tabular}

\section{Outcome and safety data}

According to Table 4, the Nebulized group has less difficult separation of CPB (12.5\% vs. $75 \%)$ and less required epinephrine ( $25 \%$ vs. $100 \%$ ). No death occurred in the Nebulized and IV group. The need for vasopressors for more than 24 hours in the IV group is more than the Nebulized group (37\% vs. 0\%). Time comparison of the extubation after ICU admission, ICU and hospital stay durations in IV group is significantly more than nebulized group and was shown in Fig. 2. 
Table 4

Outcome data.

\begin{tabular}{|llll|}
\hline Characteristic & $\begin{array}{l}\text { Nebulized group } \\
(\mathbf{n = 1 6 )}\end{array}$ & $\begin{array}{l}\text { IV group } \\
(\mathbf{n = 1 6 )}\end{array}$ & \\
\hline Difficult separation from CPB & $2(\% 12.5)$ & $12(\% 75)$ & \\
\hline Intravenous adrenaline post-CPB & $4(\% 25)$ & $16(\% 100)$ & \\
\hline Malignant arrhythmia & $2(\% 12.5)$ & 0 & \\
\hline Vasopressors use $>$ 24 hours Death & 0 & $6(\% 37)$ & \\
\hline Death & 0 & 0 & $\mathrm{P}=0.001$ \\
\hline Extubation (hour after ICU admit)(hour) & $11 \pm 5$ & $48 \pm 25$ & $\mathrm{P}=0.009$ \\
\hline ICU stay (days) & $3 \pm 1$ & $12 \pm 2$ & $\mathrm{P}=0.026$ \\
\hline Hospital stay (days) & $8 \pm 1$ & & \\
\hline $\begin{array}{l}\text { Variables expressed as number (\%) or as mean } \pm \text { SD. CPB, cardiopulmonary bypass; ICU, intensive } \\
\text { care unit. }\end{array}$ & & & \\
\hline
\end{tabular}

\section{Discussion}

The main aim of our study was to assess the effect of nebulized milrinone versus intravenous administration in patients with pulmonary hypertension who are candidates for open-heart surgery.

Our results showed that the administration of milrinone by nebulization before weaning off from CPB in cardiac surgery in patients who had pulmonary hypertension caused more stable hemodynamic (HR and MAP) and more decrease of mPAP with higher MAP/mPAP ratio than intravenous milrinone. These effects of nebulized milrinone are consistent with the previous study $(14),(7,10)$.

The patients in the two groups in our study had pulmonary hypertension, before induction and at baseline, despite this unfavorable condition, no significant systemic hypotension was observed in the nebulized milrinone group; only $25 \%$ of patients required inotropes, and none returned on CPB While in the intravenous group, $100 \%$ people needed inotropes.

The use of inhaled and intravenous milrinone in 48 patients undergoing mitral valve replacement with pulmonary hypertension were compared by Wang et al. A comparable decrease in mPAP in both groups were found, and MAP was significantly higher in the inhaled group than in the intravenous group after initiation of milrinone therapy(15).

In our study PVR in both groups decreased over time, but this decrease was more in the nebulized group. This may be due to the longer-lasting effect of inhaled medication. Denault et al. study on inhaled 
milrinone administration before surgical incision in cardiac surgery patients with pulmonary hypertension have a not significant effect on systemic hypotension, PAP, and PVR (7).

Denault et al; in another multi-center RCT were compared inhaled milrinone with placebo in cardiac surgical patients with $\mathrm{PH}$. Their study concluded that inhaled milrinone can be increase COP and reduced systolic PAP $(p=0.04)$ without reduced systemic arterial pressure or HR. In the 10 patients, The $\mathrm{MAP} / \mathrm{mPAP}$ ratio was more than $20 \%(16)$. This finding was consistent with our results.

In a meta-analysis by Rong et al, the benefits of inhaled and intravenous milrinone in adult cardiac surgery were studied. The hemodynamic and clinical effects of both methods of administration were evaluated. The researchers found that the intravenous milrinone was associated with significantly lower mPAP and SVR than control. But no significant difference between inhaled milrinone group in comparison with placebo in mPAP and SVR(17).

Lamarche et al. showed administration of a single dose of milrinone inhalation effects before and after CPB reduce reinitiation of $\mathrm{CPB}(3 \%)$ and $(23 \%)$ respectively $(10)$. The results of our research are in agreement with the above-mentioned study.

In Ibrahim et al study CHD patients with PAH undergoing on-pump cardiac surgery administration of intravenous milrinone $0.5 \mu \mathrm{g} \mathrm{kg}$ and the inhaled milrinone $50 \mu \mathrm{g} \mathrm{kg}-1$ just before weaning off CPB, showed milrinone inhalation facilitated the weaning from CPB as it significantly reduced mPAP and maintained MAP with subsequently fewer needs for vasoactive drugs(18).

In our study, the MAP/mPAP ratio as a predictor of the outcome of cardiac surgery was significantly lower in the IV group than in the nebulized milrinone group as same as previous study $(5,19,20)$. If this ratio in the preoperative period is less than 4 , it indicates a lower survival rate, more hemodynamic complications, and suggests prolong vasopressor support for more than $24 \mathrm{~h}$ or the use of intra-aortic balloon pump in patients with PH after cardiac surgery(19). The result of Ibrahim's study were consistent with our results.

In our study, the separation from CPB was easier in the nebulized milrinone group than the IV milrinone group, so the difficult separation was 1 (\% 12.5) in the nebulized milrinone group while 6 (\% 75) in the IV milrinone group and the need to epinephrine was smaller in the nebulized milrinone group than in the IV milrinone group. This effect due to systemic vasodilatation and hypotension by IV administration of milrinone. Inhaled milrinone acts only locally on pulmonary vascular beds and has minimal systemic side effects (18).

\section{Conclusion}

The major cause of milrinone administration in recent cardiac surgery methods is facilitating CPB weaning off. Because Nebulization of milrinone can more reduce PVR, and PH by the above-discussed mechanism, that can be suitable in comparison IV route. According to the obtained results, we can conclude that nebulized milrinone before weaning off from CPB, accelerated and facilitated separation 
from CPB as it significantly decreases mPAP and preserves MAP, subsequent in fewer requirements for inotrope drugs.

\section{Limitations}

Among the limitations of this research, we can refer to the small size of the sample and difficulty with following up patients after discharge for several months.

\section{Declarations}

\section{Ethics approval and consent to participate}

The present study was approved by the ethics committee of the Anesthesiology and Pain Research Center, Ahvaz Jundishapur University of Medical Sciences, Ahvaz, Iran (IR.AJUMS.REC.1399.654).

The RCT code of this study was IRCT20201104049266N1. Written informed consent signed by the all of the patients.

\section{Consent for publication}

Not applicable

\section{Availability of data and materials}

All data were retrieved from the institutional database and are available from the corresponding authors upon reasonable request.

\section{Competing interests}

The authors declare that they have no competing interests

\section{Funding}

Financial support for this study was provided by Ahvaz Jundishapur University of Medical Sciences.

(Grant No. IR.AJUMS.REC.1399.654)

\section{AUTHORS' CONTRIBUTION}

S. j. contributed in concept, study design, the definition of intellectual content; F.J.Z contributed in study design, drafted the manuscript; S.J. conceived of the study and participated in its design; M.B.Z. provided study materials and patients' information; S.J and N.B collected data. All authors read and approved the final manuscript.

\section{Acknowledgement}


This article was extracted from the thesis by Dr. Sara Joreirahmadi with Project No. PAIN-9922 and was granted by Anesthesiology and Pain Research Center of Ahvaz Jundishapur University of Medical Sciences. This study was intended as a randomized -controlled trial IRCT20201104049266N1

\section{References}

1. Kiely DG, Elliot CA, Sabroe I, Condliffe R. Pulmonary hypertension: diagnosis and management. Bmj. 2013;346.

2. Pahal P, Sharma S. Idiopathic Pulmonary Artery Hypertension (IPAH). StatPearls [Internet]. 2019.

3. Simonneau G, Gatzoulis MA, Adatia I, Celermajer D, Denton C, Ghofrani A, et al. Updated Clinical Classification of Pulmonary Hypertension. Journal of the American College of Cardiology. 2013;62(25, Supplement):D34-D41.

4. Nanayakkara S, Mak V, Crannitch K, Byrne M, Kaye DM. Extended release oral milrinone, CRD-102, for advanced heart failure. The American journal of cardiology. 2018;122(6):1017-20.

5. Nguyen AQ-N, Denault AY, Théoret Y, Perrault LP, Varin F. Inhaled milrinone in cardiac surgical patients: a pilot randomized controlled trial of jet vs. mesh nebulization. Scientific reports. 2020;10(1):1-9.

6. Hegazy N, Elhenawy A. Comparison of hemodynamic effects of inhaled milrinone and inhaled prostacyclin after adult cardiac surgery. J Appl Sci Res. 2010;6:38-44.

7. Denault A, Haddad F, Lamarche Y, Nguyen A, Varin F, Levesque S, et al. Pilot randomized controlled trial of inhaled milrinone in high-risk cardiac surgical patients. Surgery Curr Res. 2014;4(192):21611076.1000192.

8. Gong M, Lin X-Z, Lu G-T, Zheng L-J. Preoperative inhalation of milrinone attenuates inflammation in patients undergoing cardiac surgery with cardiopulmonary bypass. Medical Principles and Practice. 2012;21(1):30-5.

9. Lamarche Y, Malo O, Thorin E, Denault A, Carrier M, Roy J, et al. Inhaled but not intravenous milrinone prevents pulmonary endothelial dysfunction after cardiopulmonary bypass. The Journal of thoracic and cardiovascular surgery. 2005;130(1):83-92.

10. Lamarche Y, Perrault LP, Maltais S, Tétreault K, Lambert J, Denault AY. Preliminary experience with inhaled milrinone in cardiac surgery. European journal of cardio-thoracic surgery. 2007;31(6):1081-7.

11. Denault A, Bussières J, Arellano R, Finegan B, Gavra P, Haddad F, et al. A Multicentre RandomizedControlled Trial of Inhaled Milrinone in High-Risk Cardiac Surgical Patients. Survey of Anesthesiology. 2017;61(1):3.

12. Thunberg CA, Gaitan BD, Grewal A, Ramakrishna H, Stansbury LG, Grigore AM. Pulmonary hypertension in patients undergoing cardiac surgery: pathophysiology, perioperative management, and outcomes. Journal of cardiothoracic and vascular anesthesia. 2013;27(3):551-72.

13. Licker M, Diaper J, Cartier V, Ellenberger C, Cikirikcioglu M, Kalangos A, et al. Clinical review: management of weaning from cardiopulmonary bypass after cardiac surgery. Ann Card Anaesth. 
2012;15(3):206.

14. Hentschel T, Yin N, Riad A, Habbazettl H, Weimann J, Koster A, et al. Inhalation of the phosphodiesterase 3 inhibitor milrinone attenuates pulmonary hypertension in a rat model of congestive heart failure. The Journal of the American Society of Anesthesiologists. 2007;106(1):12431.

15. Wang $\mathrm{H}$, Gong $\mathrm{M}$, Zhou $\mathrm{B}$, Dai $\mathrm{A}$. Comparison of inhaled and intravenous milrinone in patients with pulmonary hypertension undergoing mitral valve surgery. Advances in therapy. 2009;26(4):462-8.

16. Denault AY, Bussières JS, Arellano R, Finegan B, Gavra P, Haddad F, et al. A multicentre randomizedcontrolled trial of inhaled milrinone in high-risk cardiac surgical patients. Canadian Journal of Anesthesia/Journal canadien d'anesthésie. 2016;63(10):1140-53.

17. Rong LQ, Rahouma M, Abouarab A, Di Franco A, Calautti NM, Fitzgerald MM, et al. Intravenous and inhaled milrinone in adult cardiac surgery patients: A pairwise and network meta-analysis. Journal of cardiothoracic and vascular anesthesia. 2019;33(3):663-73.

18. Abd Elbaser II, El Derie AAEA. Does Inhaled Milrinone Facilitate Weaning From Cardiopulmonary Bypass in Children with Congenital Heart Diseases Complicated with Pulmonary Arterial Hypertension? Turkish journal of anaesthesiology and reanimation. 2020;48(2):127.

19. Rebel A, Nguyen D, Bauer B, Sloan PA, DiLorenzo A, Hassan Z-U. Systemic-to-pulmonary artery pressure ratio as a predictor of patient outcome following liver transplantation. World journal of hepatology. 2016;8(32):1384.

20. Laflamme M, Perrault LP, Carrier M, Elmi-Sarabi M, Fortier A, Denault AY. Preliminary experience with combined inhaled milrinone and prostacyclin in cardiac surgical patients with pulmonary hypertension. Journal of cardiothoracic and vascular anesthesia. 2015;29(1):38-45.

\section{Figures}




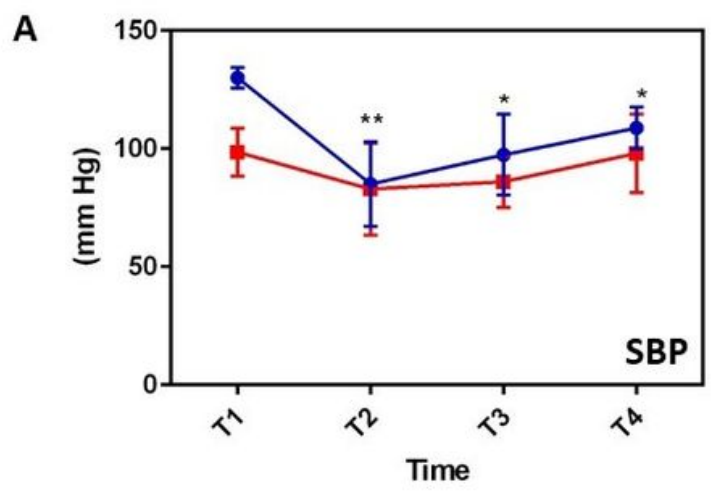

$$
\begin{aligned}
& - \text { IV group } \\
& \rightarrow \text { Nebulized group }
\end{aligned}
$$

B

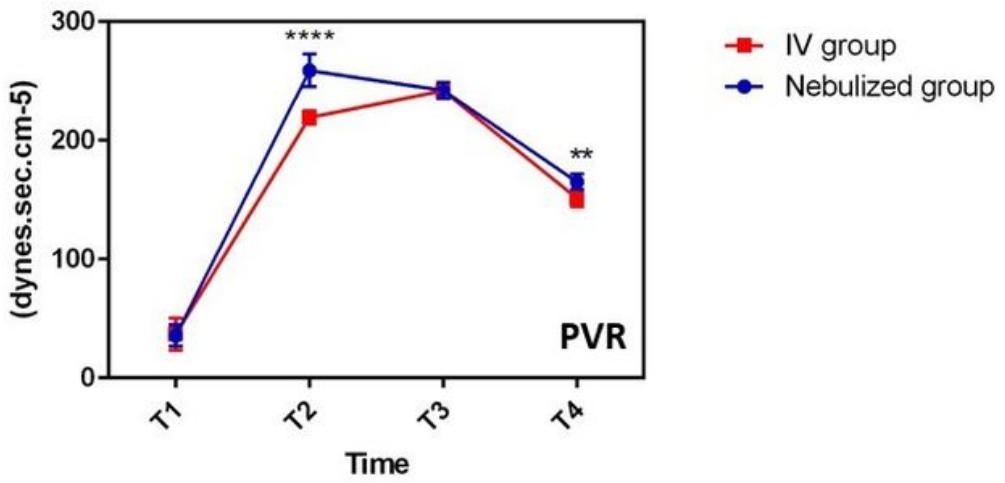

\section{Figure 1}

Comparison of the Nebulized and IV group for SBP and PVR between T2, T3, and T4. An SBP is smaller at T2, T3, and T4 in the IV group. B, PVR is higher at T2 and T4 in the Nebulized group. *: $P<0.05$ and **: $P<0.01$, $\star \star \star \star: ~ P<0.0001$ were considered as significant versus control. 

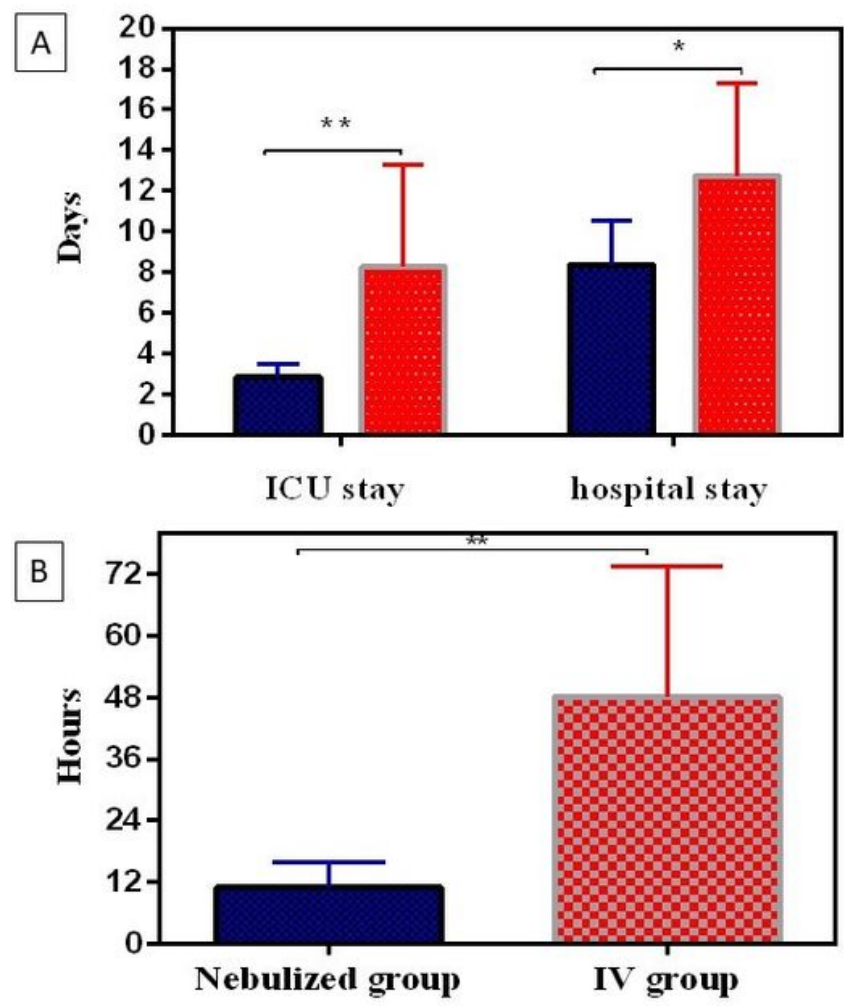

Nebulized group

13 IV group

IV group

Figure 2

Comparison of the extubation after ICU admission, ICU and hospital stay durations. A, ICU stay $(p=0.009)$ Hospital stay $(p=0.026)$ and $B$, extubation after ICU admission $(p=0.001)$ and has more time in IV group. *: $\mathrm{P}<0.05$ and **: $\mathrm{P}<0.01$ were considered as significant versus control. 


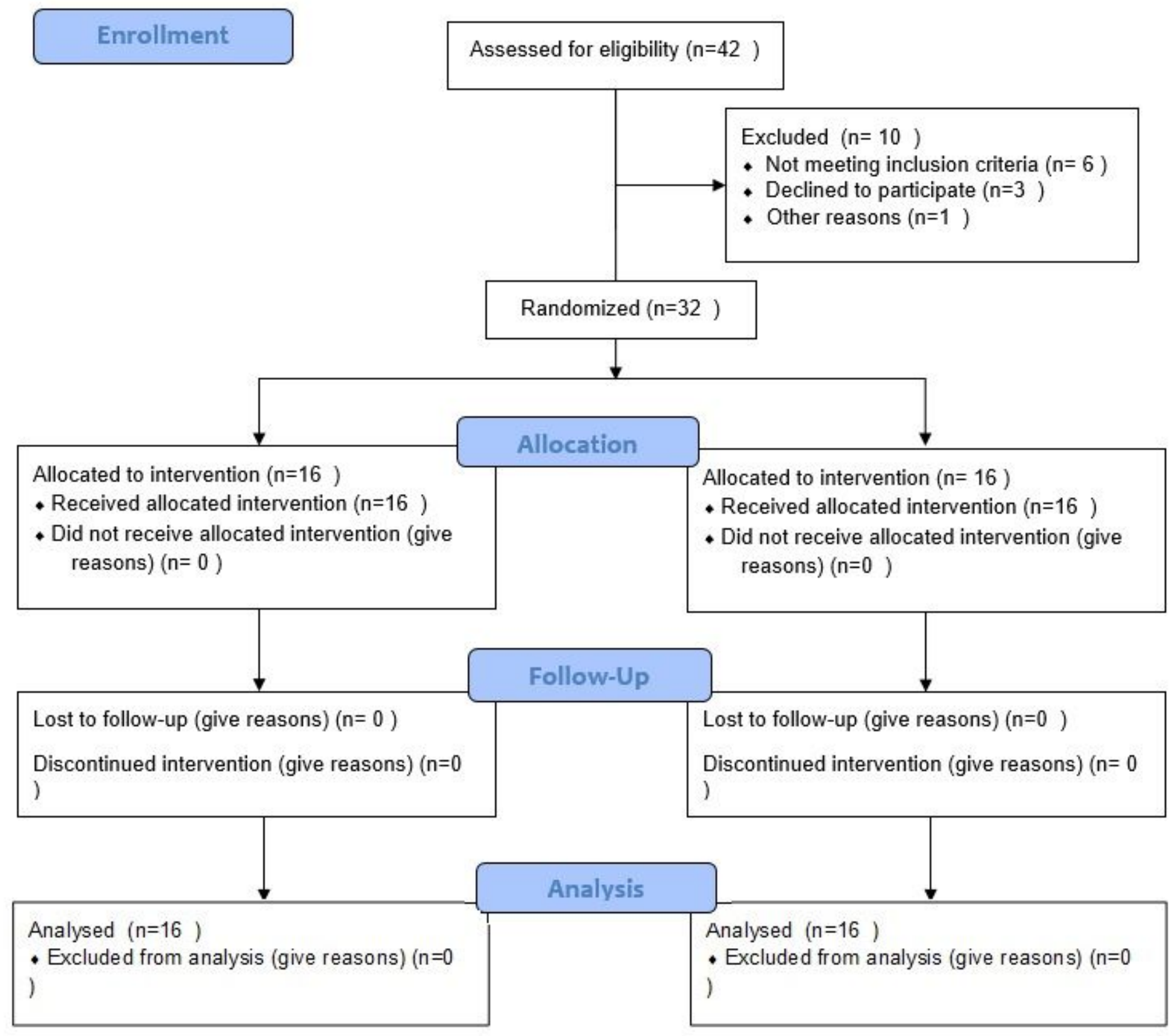

Figure 3

Consort flow diagram 